Published in final edited form as:

Ann Surg Oncol. 2019 June ; 26(6): 1737-1743. doi:10.1245/s10434-019-07263-5.

\title{
Long-Term Efficacy of Lymph Node Reoperation for Persistent Papillary Thyroid Cancer: 13-Year Follow-Up
}

\author{
Amblessed E. Onuma, MD¹, Eliza W. Beal, MD¹, Fadi Nabhan, MD², Tasha Hughes, MD $^{3}$, \\ William B. Farrar, MD ${ }^{1}$, John Phay, MD ${ }^{1}$, Matthew D. Ringel, MD², Richard T. Kloos, MD ${ }^{4}$, \\ and Lawrence A. Shirley, MD ${ }^{1}$ \\ ${ }^{1}$ Department of Surgery, Division of Surgical Oncology, The Ohio State University Wexner \\ Medical Center and James Cancer Hospital, Columbus, $\mathrm{OH}$ \\ ${ }^{2}$ Department of Internal Medicine, Division of Endocrinology, Diabetes and Metabolism, The Ohio \\ State University Wexner Medical Center and James Cancer Hospital, Columbus, $\mathrm{OH}$ \\ ${ }^{3}$ Department of Surgery, Division of Surgical Oncology, University of Michigan, Ann Arbor, MI \\ ${ }^{4}$ Department of Medical Affairs, Veracyte, Inc., South San Francisco, CA
}

\begin{abstract}
Background.-Current recommendations for persistent or recurrent locoregional papillary thyroid cancer (PTC) include consideration of surgical resection versus active surveillance. The purpose of this study is to determine long-term outcomes after surgical resection of recurrent or persistent metastatic PTC in cervical lymph nodes after failure of initial surgery and radioactive iodine therapy using newer validated clinical outcomes measures.
\end{abstract}

Methods.-Outcomes of 70 patients who underwent cervical lymphadenectomy $(n=110)$ from 1999 to 2013 for recurrent or persistent locoregional PTC metastases were reviewed. Measures included biochemical remission (BCR) based on Tg levels, American Thyroid Association classifications for response to treatment [biochemical incomplete response (BIR), structural incomplete response (SIR), indeterminate response (IR), and excellent response (ER)], need for reoperation, surgical complications, disease progression, and death.

Results.-The median follow-up was 13.1 years, with only two additional reoperations since 2010, one of which had no metastasis on pathology with the other developing anaplastic thyroid cancer in background PTC. ER was achieved in 31 (44\%) patients, all of whom remained in ER at time of last follow-up (median 14.1 years). There were no structural recurrences in patients with persistent BIR or IR after reoperation. Patients with SIR had stable disease, except for one who died due to anaplastic thyroid cancer.

Conclusions.-Patients who achieved ER after reoperation had no need for further treatment. Patients with persistent detectable Tg levels after reoperation rarely developed structural

L. A. Shirley, MD, Lawrence.Shirley@osumc.edu.

DISCLOSURE

All authors declare that they have no conflicts of interest.

Publisher's Note Springer Nature remains neutral with regard to jurisdictional claims in published maps and institutional affiliations. 
recurrence. ATA outcomes can be safely used to guide treatment decisions over a decade after reoperation for PTC.

Papillary thyroid cancer (PTC) is the most common classical endocrine malignancy, accounting for approximately $85 \%$ of all thyroid cancers, and prognosis is generally favorable, with estimated 5-year survival of 98\%. ${ }^{1,2}$ Treatment for PTC traditionally consists of total thyroidectomy with or without central neck lymph node dissection, selectively followed by radioactive iodine ablation, and thyroid stimulating hormone (TSH) suppression using levothyroxine. ${ }^{3}$ After initial treatment, surveillance includes serum thyroglobulin $(\mathrm{Tg})$ measurement and performance of neck ultrasound. ${ }^{3,4}$ In the absence of anti-thyroglobulin antibodies, Tg measurement accurately detects persistent and/or recurrent disease and, in patients undergoing TSH suppression, is highly specific. ${ }^{3-5}$ Patients with detectable Tg during TSH suppression on levothyroxine whose initial therapy included total thyroidectomy and radioactive iodine (RAI) harbor residual disease that may or may not be able to be localized structurally. ${ }^{4,5}$ For individuals with structurally persistent or recurrent disease, approximately two-thirds of the time, recurrence is localized in the cervical lymph nodes, making them candidates for surgical remission. ${ }^{6}$

Historically, it was recommended that patients with resectable locoregional PTC recurrence undergo reopera-tion. ${ }^{5}$ However, the outcomes of reoperation, whether determined by biochemical remission (BCR) or absence of structural disease, vary across studies. ${ }^{7-9}$ These variable results may be related to differences in duration of follow-up as well as in criteria used to determine postoperative disease status, including the definition of BCR. Despite several studies suggesting an indolent course for many cervical lymph node recurrences, the most recent American Thyroid Association (ATA) guidelines include consideration of surgical resection with curative intent versus active surveillance depending on size and location of persistent/recurrent locoregional disease. ${ }^{12}$

In 2010, our group completed a retrospective clinical study assessing outcomes of surgical resection of meta-static PTC in cervical lymph nodes after failure of initial total thyroidectomy and RAI therapy. Our current study aims to evaluate long-term outcomes in this same cohort after median follow-up of 13.1 years, in light of the most recent ATA guidelines.

\section{PATIENTS AND METHODS}

\section{Patients}

Ninety-five adult patients who underwent cervical lymphadenectomy at The Ohio State University Wexner Medical Center for recurrent/persistent PTC in absence of distant metastasis were identified. Patients with anti-thyroglobulin antibodies $(n=25)$ were excluded from final analysis due to inability to measure thyroglobulin levels accurately. Medical records were reviewed for demo-graphics and clinical characteristics. Measurements of disease status included serum Tg, neck ultrasound report, cross-sectional and nuclear imaging reports, and physical examination. All patients had initial total thyroidectomy (+/- nodal dissection at index operation) and at least one dose of $\mathrm{I}^{131}$ therapy. None of the patients received external-beam radiation. Surveillance included physical 
examination every 6 months, with cervical ultrasound and $\mathrm{Tg} / \mathrm{TSH}$ measurements. Patients with more worrisome features (i.e., BRAF/TERT mutation or poorly differentiated cancer) were followed every 3 months. As described in our previous publication, need for reoperation was determined based on elevated basal or stimulated Tg plus malignant cytology on fine-needle aspiration (FNA) or anatomically defined neck lesions on highresolution ultrasound (US) suspicious for recurrent PTC without FNA, palpable cervical lymphadenopathy without FNA, or positive fluo-rodeoxyglucose (FDG) positron emission tomography (PET) scan without FNA. ${ }^{13}$ Lymphadenectomy was defined as a predetermined minimum of one lymph node removed during neck reexploration. Distant metastases were excluded by preoperative chest $\mathrm{x}$-ray, low-dose $\mathrm{I}^{131}$ whole-body scintigraphy, and/or chest computed tomography (CT). A minority of patients underwent additional imaging studies such as FDG-PET, bone scan, or brain magnetic resonance imaging (MRI). The study was approved by the Ohio State University Institutional Review Board.

\section{Outcome Definitions}

Our prior publication defined biochemical remission (BCR) as undetectable Tg level after TSH stimulation via thyroid hormone withdrawal or rhTSH. ${ }^{13}$ For the current analysis, we additionally utilized measures of treatment response defined in the 2015 ATA guidelines, which were subsequently validated in several short-term stud-ies. ${ }^{7,12,14,15}$ These guidelines define four categories of disease response: (1) excellent response (ER) for negative imaging and either suppressed $\mathrm{Tg}<0.2 \mathrm{ng} / \mathrm{mL}$ or TSH-stimulated $\mathrm{Tg}<1 \mathrm{ng} / \mathrm{mL}$, (2) biochemical incomplete response (BIR) for negative imaging and suppressed $\mathrm{Tg} \geq 1 \mathrm{ng} / \mathrm{mL}$ or stimulated $\mathrm{Tg} \geq 10 \mathrm{ng} / \mathrm{mL}$ or rising anti-Tg antibody levels, (3) structural incomplete response (SIR) for structural or functional evidence of disease with any $\mathrm{Tg}$ level with or without anti-Tg antibodies, and (4) indeterminate response (IR) for nonspecific findings on imaging studies, or faint uptake in thyroid bed on RAI scanning, nonstimulated Tg detectable but $<1 \mathrm{ng} / \mathrm{mL}$, or stimulated $\mathrm{Tg}$ detectable but $<10 \mathrm{ng} / \mathrm{mL} .^{12}$

\section{Statistics}

Demographics and disease characteristics are reported as mean or median, with interquartile range (IQR) as applicable. Quantitative data were compared by Student's T test or MannWhitney U test. Descriptive analyses are used to describe patients' long-term management, recurrence, and death. All statistical analysis was performed using SPSS version 25 (SPSS, Inc., Chicago, IL, USA).

\section{RESULTS}

Seventy patients were included in the cohort. Clinicopathologic characteristics are included in Table 1 and have been previously reported. ${ }^{13}$ Median age at diagnosis was 35 years (range 15-71 years). The great majority of patients (91.4\%) had stage I disease based on AJCC VIII criteria. Median follow-up was 13.1 years (IQR 7.9-15.3 years) from time of first reoperation. 


\section{Response to Surgical Therapy}

There were 110 lymphadenectomies completed on 70 patients through April 2013. Figure 1 details the response to each reoperation. Following first reoperation, using our previous criteria, BCR was achieved in 14 (20.0\%) patients. Of these, 11 (78.6\%) remained in BCR at last follow-up. Using 2015 ATA criteria to assess response to first reoperation, 19 patients (27.1\%) achieved ER, 31 (44.3\%) had SIR, 4 (5.7\%) had BIR, and 6 (8.6\%) had IR. Importantly, all patients who achieved ER following first reoperation remained in this category at time of last follow-up (median follow-up of ER cohort 14.1 years, IQR 12.916.8 years).

Twenty-nine patients had a second reoperation: 26 for SIR, 1 for BIR, and 2 for IR. Using our previous criteria, BCR was achieved in four (13.8\%), all remaining in BCR with median follow-up of 13.2 years (IQR 9.5-14 years). Using ATA criteria, ER was achieved in 11 (37.9\%) patients and 10 (34.5\%) patients had SIR. All patients who achieved ER after second reoperation remained in this category at time of last follow-up (median 14 years, IQR 13.4-17.0 years). Six patients had a third reoperation, with one achieving ER. Two patients underwent four or more reoperations, with neither attaining ER.

Since our last publication, 52 patients were clinically assessed. After excluding 17 patients without follow-up in the most recent 2 years, 34 patients were assessed for response to therapy (Fig. 2). The majority (67.6\%) continued to demonstrate an ER, while six patients $(17.6 \%)$ remained in IR, one (2.9\%) remained in BIR, and four (11.8\%) remained in SIR. No patients currently followed have had disease progression, and none are currently undergoing treatment beyond TSH suppression.

Two patients underwent reoperation subsequent to the 2010 analysis (Table 2). One patient had achieved BCR in the initial report (patient ID 55, Table 2). She had no evidence of disease for 113 months, at which time she was noted to have a Tg level of $0.9 \mathrm{ng} / \mathrm{mL}$ with a $\mathrm{TSH}$ of $0.4 \mathrm{uIU} / \mathrm{ml}$. Neck ultrasound demonstrated suspicious right lateral cervical lymph nodes, but fine-needle aspiration was not performed. Subsequent CT, MRI, and PET scans showed no evidence of distant metastases. She then underwent right modified radical neck dissection, with pathology demonstrating nine benign lymph nodes. She currently has undetectable Tg and is categorized as ER. The second patient (patient ID 92, Table 2), who never achieved BCR after any reoperation, required a reoperation in the most recent 6 years, and eventually succumbed to anaplastic thyroid cancer in the background of previous PTC.

\section{Perioperative and Pathologic Outcomes}

The mean number of lymph nodes removed during first reoperation was 13.7 (range 1-74). The mean number removed on second reoperation was 12.8 (range 3-55). On third reoperation, the mean number of lymph nodes removed was 8.7 (range 1-34). The mean number of nodes removed was not statistically different between patients who did and did not achieve ER (11.8 versus 13.7 nodes, $p=0.47)$. Similarly, there was no statistically significant difference in the mean number of positive nodes removed between patients who did and did not achieve ER (3.4 versus 4 nodes, $p=0.31$ ). There were 10 lymphadenectomies which failed to identify recurrent PTC. Indications for these operations 
were ultrasound with FNA $(n=7)$, palpation $(n=2)$, and FDG-PET avidity $(n=1)$. After reoperations, no patient developed new permanent hypoparathyroidism, defined as persistent low serum calcium 6 months after reoperation, or permanent recurrent laryngeal nerve injury, defined as vocal cord pare-sis/paralysis on direct laryngoscopy. There was one postoperative chyle leak, which was reexplored with successful ligation of the thoracic duct.

\section{Deaths in the Cohort}

Three patients died since the time of our previous publication, with one due to thyroid cancer $(1.4 \%)$. One patient developed anaplastic thyroid cancer in the back-ground of her persistent cervical PTC metastases and died of disease. One patient underwent total thyroidectomy for PTC and 9 years later underwent right central neck dis-section for elevated $\mathrm{Tg}$, with all lymph nodes benign on final pathology. The patient was lost to followup immediately after surgery and died of unknown cause 9 years after reoperation. One patient underwent total thyroidectomy for PTC followed by bilateral modified radical neck dissection demonstrating metastatic disease in 1 of 11 lymph nodes. The patient then had persistently elevated $\mathrm{Tg}$ levels but did not have further operative explorations. Two years later, the patient died from end-stage renal disease after electing to withdraw from dialysis.

\section{DISCUSSION}

Our review of 70 patients who underwent surgical exploration for recurrent or persistent locoregional PTC disease demonstrates low rates of late recurrence and rare death related to disease with median follow-up of 13.1 years. Surgical resection of recurrent or persistent PTC in cervical lymph nodes was able to achieve BCR in 25.7\% patients (14 patients following first reoperation, and an additional 4 after second reoperation), while $44.3 \%$ of patients achieved ATA-criteria ER (19 after first reoperation, 11 after second reoperation, and 1 patient after third reoperation). No patient who achieved ER subsequently developed either structural or biochemical evidence of recurrence. Of the 34 patients currently being evaluated in clinic, over two-thirds continue to have ER, and none have required treatment beyond TSH suppression. Attaining ER may require multiple operations, but if achieved, this remission is persistent in longer-term follow-up. Despite analyzing a more aggressive cohort of PTC patients with recurrent/persistent disease, there was only one death from thyroid cancer $(1.4 \%)$.

The decision to reoperate for recurrent/persistent disease is often difficult, best conducted as a joint decision-making process between the patient and a multidisciplinary clinical team. Per recent 2015 ATA guidelines, indication for reoperation after total thyroidectomy includes rising serum Tg with biopsy-proven persistent or recurrent disease for central neck nodes $\geq 8 \mathrm{~mm}$ and lateral neck nodes $\geq 10 \mathrm{~mm}$ confirmed by anatomic imaging such as ultra-sound or CT. ${ }^{12}$ In guidelines published by Tufano et al. ${ }^{16}$, the group recommended compartmental resection for first structural recurrence, with limited/focused dissection in a previously dissected compartment, and only if disease threatened important structures. Of note, in our study, ER rates following first and second reoperations were $27.1 \%$ and $37.9 \%$ respectively, but only $16.7 \%$ after third reoperation, consistent with the recommendation of a more cautious surgical plan after initial reoperation. 
In a review of eight case series of surgical management of recurrent lymph node metastases by Urken et al., reoperation yielded BCR rates between $21 \%$ and $66 \%$, while the absence of recurrent structural disease after reoperation ranged from 51 to $100 \% .{ }^{17}$ Of note, $67.6 \%$ of patients in our study still being followed remain in ER. This is in line with the durability of ER in PTC and demonstrates that this response is durable for over a decade. Previous studies have reported that overall survival in patients with only biochemical evidence of disease was $100 \%$, compared with $85 \%$ in patients with structural evidence of persistent disease. ${ }^{16}$

Despite initial evidence of recurrent disease in all patients of our cohort, there was only one death from disease (1.4\%), confirming the rarity of death from PTC, even in those patients requiring reoperation. While survival may not differ with removing structural disease versus active surveillance, it is important to recognize additional positive impacts of achieving an ER, including reducing the intensity of follow-up, reassuring the patient, and reducing the intensity of TSH suppression. Still, prospective studies are needed to determine the optimal approach to treating patients with recurrent/persistent PTC limited to the neck.

Our study has limitations associated with retrospective cohort studies. Although the index operations for persistent or recurrent disease were performed by a single surgeon, subsequent follow-up, as well as decisions for further reoperation, were determined by several endocrinologists and surgeons. As such, there could have been a changing threshold over time to reoperate on patients with bio-chemical or structural evidence of disease. While there was a low rate of recurrence in our cohort after reoperation, it is not possible to conclude that any reoperation would necessarily change survival outcome. As this was a retrospective cohort study, there was not a matched non-surgical control group with which to compare outcomes. Finally, we recognize that the thyroglobulin assay and ultrasound technologies improved and became more sensitive over the course of the study. Thus, it is possible that some of the patients classified as BCR, or ER, may have had low but detectable Tg levels on the more sensitive assays or had identifiable structural disease using improved neck ultrasound technologies. However, these improvements would be expected to result in reduced ER and BCR rates over time, a feature not seen in our data analysis. Despite its limitations, this study is the first to demonstrate treatment durability of over a decade for selected patients requiring reoperation for PTC.

In summary, the present analysis of a reoperative thyroid cancer cohort demonstrates that the vast majority of patients who undergo surgical exploration for persistent or recurrent PTC in cervical lymph nodes remain clinically free of disease with median follow-up of 13.1 years. Once ER is achieved, it persists after more than a decade of follow-up. As well, even patients categorized as having indeterminate, biochemical incomplete, or structurally incomplete response after reoperation did not require active therapy for PTC beyond TSH suppression in the most recent 6 years of follow-up. Furthermore, none of the patients received external-beam radiation, which suggests that this treatment strategy may be unnecessary to achieve excellent outcomes even in patients at higher risk of locoregional recurrence. There was only one death from disease in this population selected for locoregional recurrence, suggesting that late development of distant metastases is either rare or occurs much later. Thus, we have demonstrated that early reoperation in this patient population has long-term durability and need for late reoperations is rare. Future prospective 
and controlled studies are needed to fully assess the efficacy of reoperation versus active surveillance in patients with locally persistent or recurrent PTC.

\section{ACKNOWLEDGEMENTS}

Funding support for this paper is through National Cancer Institute Award Number Grants P50 CA168505 (Thyroid SPORE) and P01 CA124570 from the National Cancer Institute, Award Number Grant KL2TR002734 from the National Center For Advancing Translational Sciences, and Award Number Grant 1T32AI106704-01A1 (Advanced Research Training in Immunology for Surgery Trainees) from the National Institute of Allergy and Infectious Diseases. The research reported in this publication and the content is solely the responsibility of the authors and does not necessarily represent the official views of the National Institutes of Health.

\section{REFERENCES}

1. Nguyen QT, Lee EJ, Huang MG, Park YI, Khullar A, Plodkowski RA. Diagnosis and treatment of patients with thyroid cancer. Am Health Drug Benefits 2015;8(1):30-40.

2. Cancer Facts and Figures 2018 https://www.cancer.org/content/dam/cancer-org/research/cancerfacts-and-statistics/annual-cancer-facts-and-figures/2018/cancer-facts-and-figures-2018.pdf. Accessed 27 August 2018.

3. Kloos RT. Papillary thyroid cancer: medical management and follow-up. Curr Treat Options Oncol 2005;6(4):323-338. [PubMed: 15967085]

4. Kloos RT, Mazzaferri EL. A single recombinant human thyrotropin-stimulated serum thyroglobulin measurement predicts differentiated thyroid carcinoma metastases three to five years later. J Clin Endocrinol Metab 2005;90(9):5047-5057. [PubMed: 15972576]

5. Cooper DS, Doherty GM, Haugen BR, et al. Revised American Thyroid Association management guidelines for patients with thyroid nodules and differentiated thyroid cancer. Thyroid 2009;19(11): 1167-1214.. [PubMed: 19860577]

6. Schlumberger MJ. Papillary and follicular thyroid carcinoma. N Engl J Med 1998;338(5):297-306. [PubMed: 9445411]

7. Tuttle RM, Tala H, Shah J, et al. Estimating risk of recurrence in differentiated thyroid cancer after total thyroidectomy and radioactive iodine remnant ablation: using response to therapy variables to modify the initial risk estimates predicted by the new American Thyroid Association staging system. Thyroid 2010;20(12):1341-1349. [PubMed: 21034228]

8. Yim JH, Kim WB, Kim EY, et al. The outcomes of first reoperation for locoregionally recurrent/ persistent papillary thyroid carcinoma in patients who initially underwent total thyroidectomy and remnant ablation. J Clin Endocrinol Metab 2011; 96(7):2049-2056. [PubMed: 21508143]

9. Schuff KG, Weber SM, Givi B, Samuels MH, Andersen PE, Cohen JI. Efficacy of nodal dissection for treatment of persistent/ recurrent papillary thyroid cancer. Laryngoscope 2008;118(5): 768-775. [PubMed: 18197134]

10. Rondeau G, Fish S, Hann LE, Fagin JA, Tuttle RM. Ultrasono-graphically detected small thyroid bed nodules identified after total thyroidectomy for differentiated thyroid cancer seldom show clinically significant structural progression. Thyroid 2011;21(8): 845-853. [PubMed: 21809914]

11. Robenshtok E, Fish S, Bach A, DomInguez JM, Shaha A, Tuttle RM. Suspicious cervical lymph nodes detected after thyroidectomy for papillary thyroid cancer usually remain stable over years in properly selected patients. J Clin Endocrinol Metab 2012; 97(8):2706-2713. [PubMed: 22639292]

12. Haugen BR, Alexander EK, Bible KC, et al. 2015 American Thyroid Association management guidelines for adult patients with thyroid nodules and differentiated thyroid cancer: The American Thyroid Association Guidelines Task Force on Thyroid Nodules and Differentiated Thyroid Cancer. Thyroid 2016;26(1):1-133. [PubMed: 26462967]

13. Al-Saif O, Farrar WB, Bloomston M, Porter K, Ringel MD, Kloos RT. Long-term efficacy of lymph node reoperation for persistent papillary thyroid cancer. J Clin Endocrinol Metab 2010;95(5):2187-2194. [PubMed: 20332244] 
14. Vaisman F, Momesso D, Bulzico DA, et al. Spontaneous remission in thyroid cancer patients after biochemical incomplete response to initial therapy. Clin Endocrinol (Oxf) 2012;77(1): 132-138. [PubMed: 22248037]

15. Lee SG, Lee WK, Lee HS, et al. Practical Performance of the 2015 American Thyroid Association guidelines for predicting tumor recurrence in patients with papillary thyroid cancer in South Korea. Thyroid 2017;27(2):174-181. [PubMed: 27750028]

16. Tufano RP, Clayman G, Heller KS, et al. Management of recurrent/persistent nodal disease in patients with differentiated thyroid cancer: a critical review of the risks and benefits of surgical intervention versus active surveillance. Thyroid 2015; 25(1):15-27. [PubMed: 25246079]

17. Urken ML, Milas M, Randolph GW, et al. Management of recurrent and persistent metastatic lymph nodes in well-differentiated thyroid cancer: a multifactorial decision-making guide for the Thyroid Cancer Care Collaborative. Head Neck 2015; 37(4):605-614. [PubMed: 24436291] 


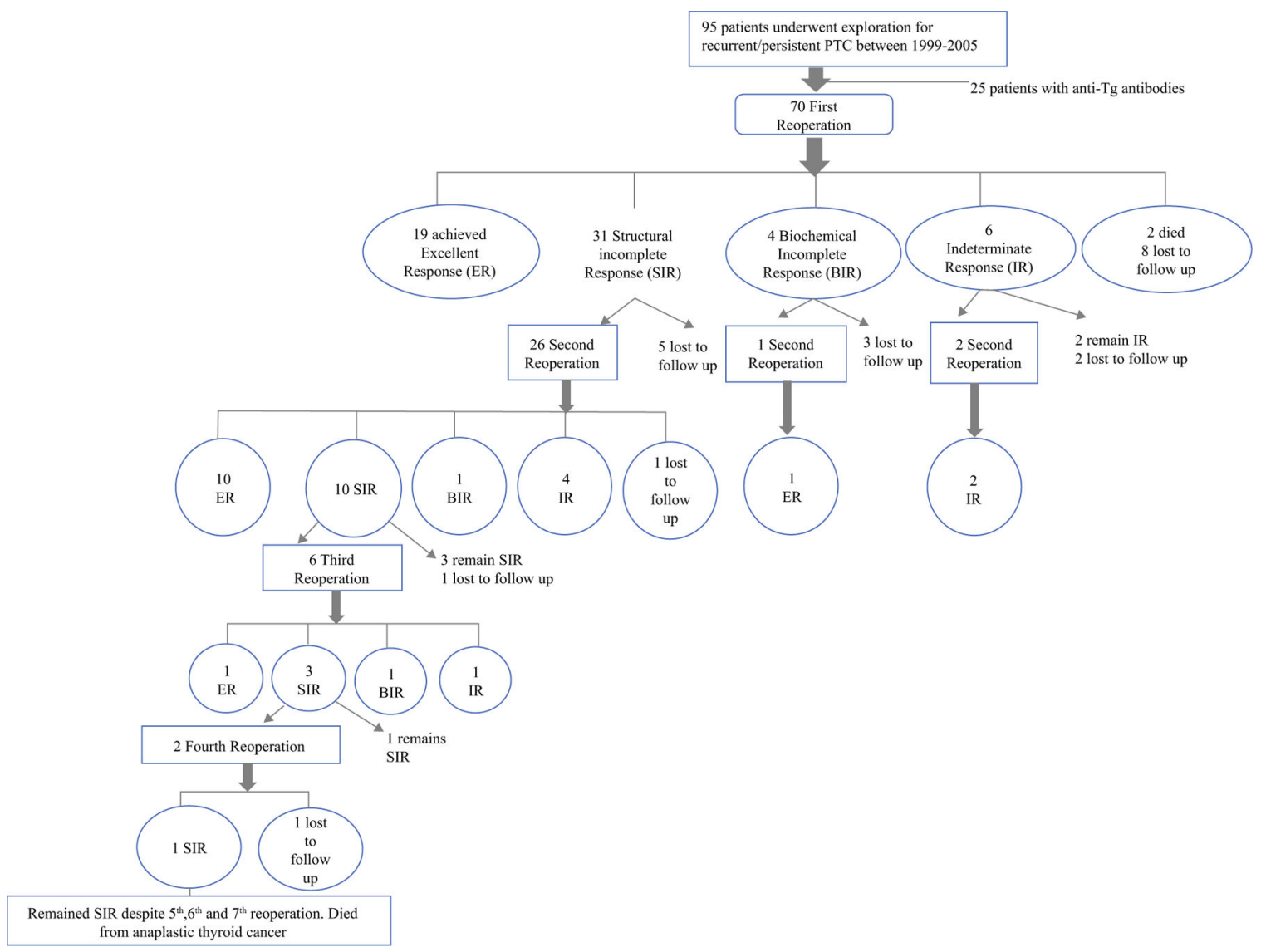

FIG. 1.

Patient response, as defined by 2015 American Thyroid Association guidelines, after each reoperation for locoregional recurrence of papillary thyroid cancer 


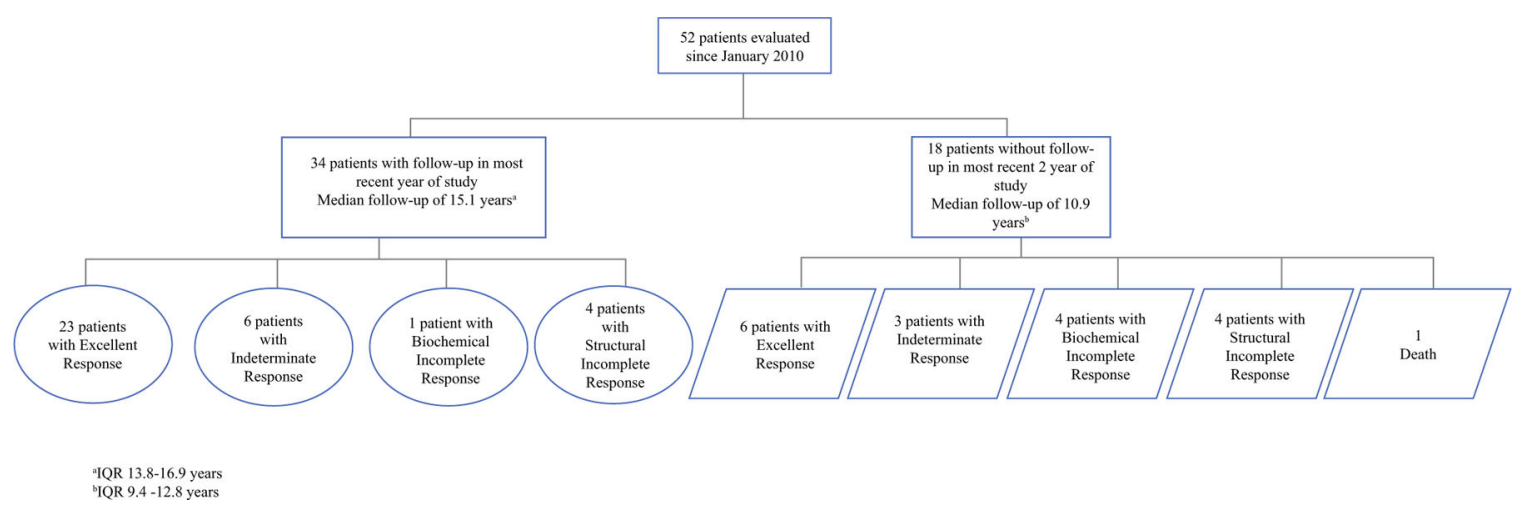

FIG. 2.

Patient response, as defined by 2015 American Thyroid Association guidelines, at time of most recent follow-up. IQR Interquartile range 


\section{TABLE 1}

\section{Clinicopathologic characteristics}

\begin{tabular}{|c|c|}
\hline Variable & Number/median (range) \\
\hline Male & 22 \\
\hline Female & 48 \\
\hline Age at diagnosis of PTC (years) & Median $35(15-71)$ \\
\hline Age at first neck reexploration (years) & Median 41 (18-73) \\
\hline Time from first thyroidectomy to first neck reexploration (years) & Median $3(<1-24)$ \\
\hline \multicolumn{2}{|l|}{ Age (years) } \\
\hline$\leq 40$ years & 48 \\
\hline$>40$ years & 22 \\
\hline \multicolumn{2}{|l|}{ AJCC VIII stage at thyroidectomy } \\
\hline I & 64 \\
\hline II & 2 \\
\hline III & 4 \\
\hline \multicolumn{2}{|l|}{ Tumor size } \\
\hline$<4 \mathrm{~cm}$ & 61 \\
\hline$>4 \mathrm{~cm}$ & 4 \\
\hline Unknown & 5 \\
\hline \multicolumn{2}{|l|}{ Capsular invasion } \\
\hline Yes & 42 \\
\hline No & 24 \\
\hline Unknown & 4 \\
\hline \multicolumn{2}{|l|}{ Lymph node status at thyroidectomy } \\
\hline Positive & 52 \\
\hline Normal & 16 \\
\hline Unknown & 2 \\
\hline Postoperative radioactive iodine $(\mathrm{mCi})$ & Median 217 (29-995) \\
\hline
\end{tabular}

Adopted from Al-Saif et al. ${ }^{13}$ and verified with patients' updated medical records 


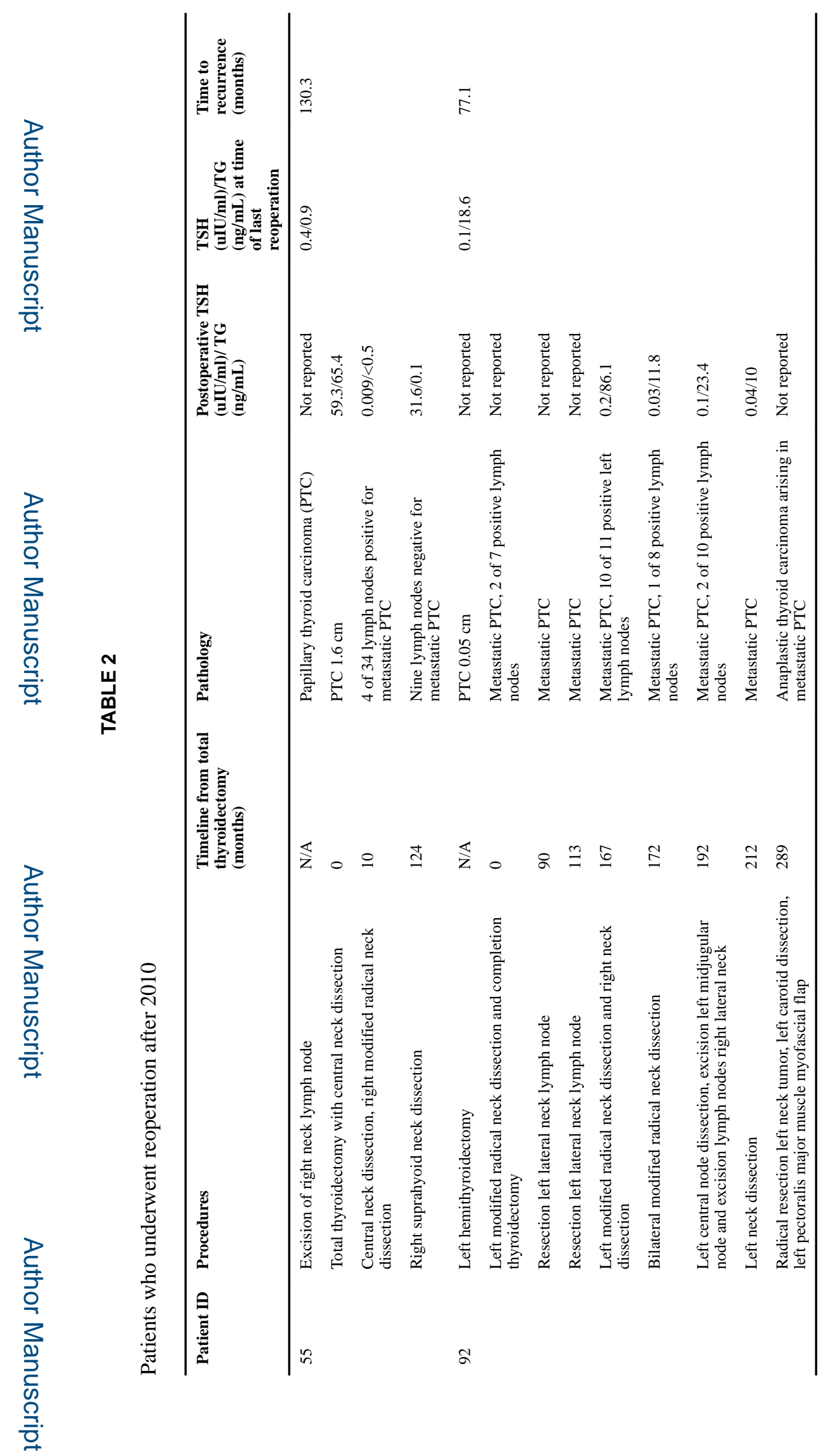

Ann Surg Oncol. Author manuscript; available in PMC 2020 June 01. 